\title{
Laboratory prediction of primary postpartum haemorrhage: a comparative cohort study
}

\author{
William K. B. A. Owiredu", Derick N. M. Osakunor ${ }^{1}$, Cornelius A. Turpin² and Osei Owusu-Afriyie ${ }^{1}$
}

\begin{abstract}
Background: Postpartum haemorrhage (PPH) is the leading cause of maternal deaths, the world over. The aim of this study was to determine laboratory parameters that could serve as risk factors for primary PPH.

Methods: This comparative cohort study involved 350 pregnant women at term who were recruited consecutively from the Komfo Anokye Teaching Hospital, Kumasi, Ghana. PPH was defined as a measured blood loss $\geq 500 \mathrm{ml}$ or enough to cause haemodynamic shock. Basic demographic data was gathered and blood was collected for laboratory assays before delivery. Univariate and multivariate logistic regression models were used to identify variables that were significantly associated with primary PPH.

Results: Of the total recruited study participants (350), five declined to participate and 74 went through caesarean section, episiotomy or instrumental deliveries and were excluded. Of the remaining (271) study participants who went through spontaneous vaginal delivery, fifty five (55) were diagnosed with primary PPH (Group 1) and the remaining 216 were those who did not have PPH (Group 2). Demographic characteristics did not differ between the two groups $(P>0.05)$. Univariate analysis showed that AST $(P=0.043)$, urea $(P<0.001)$, creatinine $(P=0.002)$, urea-to-creatinine ratio $(P=0.014)$ and the proportion of abnormal peripheral blood smear $(P<0.001)$ was higher among women in Group 1 compared to those in Group 2. Women in Group 1 had a significantly lower haemoglobin concentration (10.7 g/dL) compared to those in Group $2(12.1 \mathrm{~g} / \mathrm{dL})$. Upon multivariate analysis, an abnormal peripheral blood smear $(A O R=2.9672), H b,(A O R=0.5791)$, moderate to severe anaemia $(H b<10 \mathrm{~g} / \mathrm{dL})(A O R=3.1385)$, Urea $(A O R=$ 3.6435) and intra-renal azotaemia $(A O R=0.1893)$ remained significant.
\end{abstract}

Conclusion: Many laboratory parameters are associated with primary PPH but only a few are independent risk factors. A total clinical work-up including laboratory evaluation of the independent blood variables identified in this study will help a great deal to identify individuals at high risk for PPH.

Keywords: Haemoglobin (anaemia), Laboratory, Postpartum haemorrhage, Prediction, Risk factors, Urea

\section{Background}

Each year, about 289,000 women worldwide, die from various complications of pregnancy and childbirth and developing countries account for $99 \%(286,000)$ of this rate [1]. In 2013, Ghana recorded a maternal mortality rate of 380 per 100,000 live births [2]. Among the various causes of maternal mortality,

\footnotetext{
* Correspondence: wkbaowiredu@yahoo.com

${ }^{1}$ Department of Molecular Medicine, School of Medical Sciences (SMS), Kwame Nkrumah University of Science and Technology (KNUST), Kumasi, Ghana

Full list of author information is available at the end of the article
}

postpartum haemorrhage (PPH) has historically been the single most important [3].

Primary PPH is defined as a loss of $500 \mathrm{ml}$ or more of blood (or any amount that causes haemodynamic compromise) during and after childbirth, within the first twenty-four hours [4]. The condition presents with several consequences, which could transform a healthy pregnant woman in labour to a critically ill patient [5], and efforts to save both mother and baby may pose serious challenges to health workers [6].

Although the burden of primary PPH is severe, only few studies in the developing world, have examined 
laboratory risk factors associated with primary PPH. Risk factors associated with primary $\mathrm{PPH}$, as reported by other studies include non-white ethnicity [7], older age [8], history of PPH [9], history of blood disorders [7], nulliparity [10], low parity [11], grand multiparity [12], high blood pressure [13], antepartum haemorrhage [14] and multiple pregnancies [8].

However, many of these factors have a low predictive value [13]. Reports show that prediction of PPH with the current antenatal screenings is poor and about $60 \%$ of cases have no identifiable risk $[15,16]$. Other studies have shown that in women with three or more risk factors, predictive ability is as low as $10 \%$ [17], with Tsu's long-standing 1993 study reporting a prediction rate of below $7 \%$ [18]. Furthermore, most reports on risk factors for PPH were conducted in the developed world, where conditions, facilities, expertise and even the experience of patients may vary from that in the developing world. This is supported by reports on the role of intrapartum factors - of which the above factors play a vital role, in the development of primary $\mathrm{PPH}[7,9,12$, $13,19,20]$. With the huge burden associated with primary $\mathrm{PPH}$ there is a need for continued examination of risk factors to provide better opportunities for prevention, intervention or active management in the early stages.

The main objective of this study was to determine laboratory parameters that could serve as risk factors for primary PPH. We hypothesized that various antepartum laboratory parameters will be associated with primary $\mathrm{PPH}$ and may be of value in the clinical management of pregnant women during labour.

\section{Methods}

\section{Study design/Site}

This was a comparative cohort study with a consecutive sampling technique, carried out from April to October 2012. Three hundred and fifty (350) women who had been admitted to the labour ward of the Department of Obstetrics and Gynaecology, Komfo Anokye Teaching Hospital (KATH), Kumasi, Ghana, were recruited for the study. This was done under the supervision of qualified midwives and Obstetrician/Gynaecologists.

\section{Eligibility}

To qualify for recruitment, the pregnant women had to be on admission at the labour ward, with singleton pregnancy and should have consented to participate in the study. Women of all parity were included.

Women who underwent planned or emergency caesarean section, episiotomy and instrumental deliveries were excluded. Patients who bled after initial blood volumes had been estimated were also excluded. Women with pregnancy induced hypertension or pre-eclampsia, chronic metabolic diseases of which they were being treated or managed (e.g. diabetes mellitus, chronic kidney disease), hepatitis, HIV and tuberculosis were excluded. Inclusion was ascertained through review of patient folders in consultation with attending physicians.

\section{Participants}

Three hundred and fifty (350) pregnant women who had been admitted to the labour ward of the Department of Obstetrics and Gynaecology for delivery were recruited as a start cohort. All recruited participants were followed up until delivery and an attending Obstetrician/Gynaecologist did an appropriate assessment of clinical status/ diagnosis.

Five of the eligible recruits declined to participate in the study whereas 74 other patients underwent caesarean section, episiotomy or instrumental delivery or were lost to follow up. Of the 271 women who had normal vaginal delivery, 55 developed primary PPH (Group 1) and 216 did not develop primary PPH - normal vaginal delivery (Group 2).

\section{Sample and data collection}

Prior to delivery, about $9 \mathrm{ml}$ venous blood sample was collected from each participant into sodium citrate tubes for coagulation studies (Prothrombin Time - PT and activated Partial Thromboplastin Time - aPTT), serum separator tubes for biochemical assays (Total protein- TP, Albumin - ALB, Globulins-GLOB, Alanine Aminotransferase - ALT, Aspartate Aminotransferase - AST, urea -URE and creatinine - CRE) and ethylenediaminetetraacetic acid tubes (EDTA) tubes for haematological assays (Full Blood Count - FBC, Erythrocyte Sedimentation Rate - ESR, Glucose-6phosphate Dehydrogenase - G6PD, sickling, blood group and peripheral blood smear).

Haematological assays were performed on fresh anticoagulated blood using an automated analyser (Mindray BC 3000 Plus, Shenzhen, China). Serum separator and sodium citrated samples were centrifuged and aliquots of plasma or serum were stored at $-80^{\circ} \mathrm{C}$ until assayed. Biochemical assays were performed using an automated analyser (Selectra Pro S, Vital Scientific NV, Netherlands). Coagulation parameters were assayed using a semi-automated analyser (Coatron M4 TECO Medical Instruments, Germany). All other parameters were gathered from the patient records.

\section{Measurement of blood loss}

Deliveries were done on a dry disposable "linen saver" as suggested in a similar study [21]. The amniotic fluid was allowed to drain away and blood was allowed to collect into a graduated receptacle placed below the delivery 
bed, after which a qualified Midwife measured the amount of blood loss. Diagnosis of primary PPH (by an attending physician-Obstetrician/Gynaecologist) was based on vaginal delivery with a measured blood loss of $500 \mathrm{ml}$ or more, up to one hour post-delivery. Vaginal delivery with a measured blood loss of less than $500 \mathrm{ml}$ but enough to cause signs of haemodynamic compromise (e.g. weakness, syncope, decreased level of consciousness, dizziness, chest discomfort, dyspnoea, hypotension diaphoresis and pulmonary congestion) also constituted primary $\mathrm{PPH}[4,5,8,22]$.

\section{Outcome criteria}

All laboratory results were classified into various categories based on reference ranges with respect to pregnancy to accommodate the physiological changes [23].

In the peripheral blood smear, any feature consistent with the presence of abnormal number, shape, size and age of cells constituted an abnormal peripheral blood smear.

Classification of anaemia was as per the recommendations of the World Health Organization [24]. Azotaemia was classified as pre-renal when urea: creatinine ratio was $>100$, post-renal when ratio was from $40-100$ and intra-renal when ratio was $<40$.

\section{Statistics}

Data was entered into a Microsoft Excel spreadsheet and statistical analysis was done using the R-console (The R foundation for statistical computing, http://www.R-pro ject.org). We reported categorical variables as frequencies and proportions and compared them with Chisquare and Fisher's exact test as appropriate. We reported continuous variables as mean (SD) or median (IQR) based on data distribution (normal or skewed respectively) and compared them using WilcoxonMann-Whitney (Rank sum) test and Independent samples $t$-test for skewed and normally distributed data respectively. Multivariate logistic regression analysis was done to identify independent risk factors associated with primary PPH. Independent risk factors were identified using an automatic selection process (MODELSTEP), which removes non-significant variables and confounders in a stepwise backward selection process, based on a likelihood ratio static. An Akaike's Information Criterion (AIC) is constantly generated and the last MODELSTEP with the smallest AIC was selected as the best-fit model. Due to the low numbers of some blood types observed, this variable was categorised into "Blood type O" and "non-O blood types".

\section{Ethical Consent}

The study was approved by the Committee on Human Research Publication and Ethics (CHRPE) of the Kwame
Nkrumah University of Science and Technology (KNUST)/ School of Medical Sciences (SMS) as well as the Research and Development unit of the KATH. The objective of the study was explained to all participants and written informed consent was obtained before recruitment.

\section{Results}

Of the total number of participants approached (350), five declined to participate and a further 74 were excluded from data analysis and presentation due to selection criteria used in the present study. Of the remaining 271 study participants who went through spontaneous vaginal delivery, fifty five (55) were diagnosed with primary PPH (Group 1) where as two hundred and sixteen (216) did not present with any such complication (Group 2).

The mean age of participants was $27.7 \pm 5.7$ years and Groups 1 and 2 did not differ based on age, BMI, parity, gravidity, history of antepartum bleeding, induced and spontaneous abortions, transfusions, contraception, fibroids and surgery $(P>0.05)$.

Table 1 shows a list of biochemical and coagulation parameters of the study population. AST $(P=0.043)$, urea $(P<0.001)$, creatinine $(P=0.002)$ and Urea-tocreatinine ratio $(P=0.014)$ levels were higher amongst women in Group 1 than in Group 2.

Women in Group 1 had a significantly lower $\mathrm{Hb}(10.7$ $\mathrm{g} / \mathrm{dL})$ compared to $12.1 \mathrm{~g} / \mathrm{dL}$ in Group $2(P<0.001)$. A larger proportion of Group 1 participants had moderate/ severe anaemia in addition to an abnormal peripheral blood smear, than in Group $2(P<0.001$ each). Total WBC, NEUT, MONO and ESR levels were higher amongst women in Group 1 compared to Group $2(P<$ $0.05)$. Laboratory-confirmed presence of malaria parasitaemia was low $(6.3 \%)$ in the total population. [Tables 2 and 3]

Upon multivariate analysis, five variables remained significant. An abnormal peripheral blood smear was associated with the development of primary PPH $(\mathrm{AOR}=$ 2.9672). For every unit increase in $\mathrm{Hb}$, the odds of developing primary $\mathrm{PPH}$ decreased 0.5791 times $(95 \% \mathrm{CI}=$ 0.4585 to 0.7314 ) and women with moderate to severe anaemia $(\mathrm{Hb}<10 \mathrm{~g} / \mathrm{dL})$ were three times more likely to develop primary PPH than women with no or mild anaemia. For every unit increase in serum urea, the odds of primary PPH increased 3.6435 times. Intra-renal azotaemia was associated with a less likelihood of developing primary $\mathrm{PPH} \quad(\mathrm{AOR}=0.1893,95 \% \mathrm{CI}=$ 0.0602 to 0.5955 ) compared to pre-renal or post-renal azotaemia. [Table 4]

\section{Discussion}

We hypothesized that various antepartum laboratory parameters will be associated with primary $\mathrm{PPH}$ and will 
Table 1 Biochemical and coagulation parameters of study participants

\begin{tabular}{|c|c|c|c|c|}
\hline Parameters & All women $(n=271)$ & Group $1(n=55)$ & Group $2(n=216)$ & $P$ value \\
\hline AST median (IQR) & $17(13.5,21)$ & $18(15,22.5)$ & $16(13,20)$ & 0.043 \\
\hline ALT median (IQR) & $9(6,11)$ & $8(6.5,11)$ & $9(6,11)$ & 0.48 \\
\hline TP median (IQR) & $68(63,73)$ & $66(59.5,74)$ & $68(63,73)$ & 0.315 \\
\hline ALB median (IQR) & $36(34,38)$ & $35(31.5,38)$ & $37(35,39)$ & 0.001 \\
\hline GLOB median (IQR) & $30(28,34)$ & $31(27.5,36)$ & $30(28,34)$ & 0.889 \\
\hline URE median (IQR) & $1.8(1.5,2.4)$ & $2.5(1.8,3.2)$ & $1.8(1.5,2.3)$ & $<0.001$ \\
\hline CRE median (IQR) & $66(52.5,78.5)$ & $72(61,90.5)$ & $61.5(51,75.2)$ & 0.002 \\
\hline \multicolumn{5}{|l|}{ URE: CRE } \\
\hline Median (IQR) & $28(22,38)$ & $31(24,45)$ & $27(21,36.8)$ & 0.014 \\
\hline Intra-renal azotaemia $n(\%)$ & $217(80.7)$ & 35 (63.6) & $182(85)$ & $<0.001$ \\
\hline Normal n (\%) & $50(18.6)$ & $18(32.7)$ & $32(15)$ & \\
\hline Pre-renal azotaemia $n(\%)$ & $2(0.7)$ & $2(3.6)$ & $0(0)$ & \\
\hline PT median (IQR) & $13(12,15)$ & $14(13,16)$ & $13(12,15)$ & 0.096 \\
\hline INR median (IQR) & $1.1(1,1.3)$ & $1.2(1.1,1.4)$ & $1.1(1,1.3)$ & 0.096 \\
\hline aPTT median (IQR) & $28(24,36)$ & $31(24,38)$ & $28(24.8,33.5)$ & 0.576 \\
\hline
\end{tabular}

Data are presented as $n(\%)$ or median (IQR). All P values less than 0.05 were considered significant. Group 1: Women with primary PPH. Group 2: Women without primary PPH. IQR: Inter-quartile range. n: Number. AST: Aspartate Transaminase. ALT: Alanine Transaminases. TP: Total Protein. ALB: Albumin. GLOB: Globulins. URE: Urea. CRE: Creatinine. PT: Prothrombin Time. aPTT: Activated Partial Thromboplastin Time. INR: International Normalised Ratio

Table 2 Haematological parameters of study participants

\begin{tabular}{|c|c|c|c|c|}
\hline Parameters & All women $(n=271)$ & Group $1(n=55)$ & Group $2(n=216)$ & $P$ value \\
\hline $\mathrm{Hb}(\mathrm{g} / \mathrm{dL})$ median $(I \mathrm{QR})$ & $11.9(10.9,12.8)$ & $10.7(8.5,12.3)$ & $12.1(11.3,13)$ & $<0.001$ \\
\hline \multicolumn{5}{|l|}{ ANAEMIA $n(\%)$} \\
\hline Mod-Sev & $36(13.3)$ & $22(40)$ & $14(6.5)$ & \multirow[t]{3}{*}{$<0.001$} \\
\hline Mild & $37(13.7)$ & $9(16.4)$ & $28(13)$ & \\
\hline Normal & $198(73.1)$ & $24(43.6)$ & $174(80.6)$ & \\
\hline \multicolumn{5}{|l|}{ G6PD $n$ (\%) } \\
\hline Full Defect & $12(4.4)$ & $0(0)$ & $12(5.6)$ & \multirow[t]{3}{*}{0.169} \\
\hline No Defect & $257(94.8)$ & $55(100)$ & $202(93.5)$ & \\
\hline Partial Defect & $2(0.7)$ & $0(0)$ & $2(0.9)$ & \\
\hline \multicolumn{5}{|l|}{ SICKLING n (\%) } \\
\hline Negative & $235(86.7)$ & $47(85.5)$ & $188(87)$ & \multirow[t]{2}{*}{0.931} \\
\hline Positive & $36(13.3)$ & $8(14.5)$ & $28(13)$ & \\
\hline \multicolumn{5}{|l|}{ BLOOD GROUP n (\%) } \\
\hline Non-O groups & $130(47.9)$ & $22(40)$ & $108(50)$ & \multirow[t]{2}{*}{0.227} \\
\hline Group O & $141(52.1)$ & $33(60)$ & $108(50)$ & \\
\hline HCT median (IQR) & $36.1(32.9,38.5)$ & $32.1(25.2,36.8)$ & $36.5(33.6,38.5)$ & $<0.001$ \\
\hline MCV median (IQR) & $84.8(80.5,88.6)$ & $85.9(81.6,88.2)$ & $84(80.1,88.7)$ & 0.426 \\
\hline $\mathrm{MCH}$ median (IQR) & $28.1(26.9,29.7)$ & $29(27.4,30.1)$ & $28.1(26.8,29.6)$ & 0.151 \\
\hline MCHC median (IQR) & $33.2(32.6,34.3)$ & $33.4(32.8,34.3)$ & $33.2(32.6,34.3)$ & 0.248 \\
\hline PLT mean (SD) & $205.8(66.8)$ & $210(69.3)$ & 204.7 (66.2) & 0.597 \\
\hline
\end{tabular}

Data are presented as $\mathrm{n}(\%)$ or median (IQR) or mean (SD). All $P$ values less than 0.05 were considered significant. Group 1: Women with primary PPH. Group 2: Women without primary PPH. IQR: Inter-quartile range. n: Number. Mod: Moderate. Sev: Severe. Hb: Haemoglobin. G6PD: Glucose-6-Phosphate Dehydrogenase. HCT: Haematocrit. MCV: Mean Cell Volume. MCH: Mean cell haemoglobin. MCHC: Mean Corpuscular Haemoglobin Concentration. PLT: Platelets 
Table 3 Haematological parameters of study participants

\begin{tabular}{|c|c|c|c|c|}
\hline Parameters & All women $(n=271)$ & Group $1(n=55)$ & Group $2(n=216)$ & $P$ value \\
\hline \multicolumn{5}{|l|}{ WBC median (IQR) } \\
\hline Total & $9.4(7.4,12.9)$ & $10.2(8.8,15)$ & $9.4(7.1,12.5)$ & 0.023 \\
\hline NEUT & $7.3(5.1,11)$ & $8.6(6,12.9)$ & $7(4.8,9.8)$ & 0.004 \\
\hline LYMPH & $1.5(1.2,2)$ & $1.6(1.2,1.9)$ & $1.5(1.2,2)$ & 0.831 \\
\hline MONO & $0.7(0.6,0.9)$ & $0.8(0.5,1.2)$ & $0.7(0.6,0.9)$ & 0.017 \\
\hline EO & $0(0,0.1)$ & $0(0,0.1)$ & $0(0,0.1)$ & 0.116 \\
\hline BASO & $0(0,0)$ & $0(0,0)$ & $0(0,0)$ & 0.119 \\
\hline ESR (mm/hr) median (IQR) & $56(39.5,76)$ & $70(48,96)$ & $55(37,75)$ & 0.018 \\
\hline \multicolumn{5}{|c|}{ MALARIA PARASITAEMIA $n(\%)$} \\
\hline Negative & $254(93.7)$ & $51(92.7)$ & $203(94)$ & 0.756 \\
\hline Positive & $17(6.3)$ & $4(7.3)$ & $13(6)$ & \\
\hline \multicolumn{5}{|c|}{ PERIPHERAL BLOOD SMEAR n (\%) } \\
\hline Abnormal & $99(36.5)$ & $39(70.9)$ & $60(27.8)$ & $<0.001$ \\
\hline Normal & $172(63.5)$ & $16(29.1)$ & $156(72.2)$ & \\
\hline
\end{tabular}

Data are presented as $\mathrm{n}$ (\%) or median (IQR) or mean (SD). All P values less than 0.05 were considered significant. Group 1: Women with primary PPH. Group 2: Women without primary PPH. IQR: Inter-quartile range. N: Number. WBC; White Blood Cell. NEUT: neutrophils. LYMPH: Lymphocyte. MONO: Monocytes. EO: Eosinophil. BASO: Basophil. ESR: Erythrocyte Sedimentation Rate

have the ability for identification of high-risk groups. The results of our study confirm this hypothesis but only few of the parameters included in the present study showed independent association. A number of factors showed an association with primary $\mathrm{PPH}$; AST, urea, creatinine, urea-to-creatinine ratio, haemoglobin concentration, WBC, ESR and the state of a peripheral blood smear (normal or abnormal). Multivariate analysis showed that increasing urea and ureato-creatinine ratio, decreasing haemoglobin concentration

Table 4 Multivariate analysis for independent factors associated with primary PPH

\begin{tabular}{llll}
\hline Parameter & Adj OR & $95 \% \mathrm{Cl}$ & $P$ value \\
\hline $\begin{array}{l}\text { Peripheral blood smear } \\
\text { *Normal }\end{array}$ & & & \\
Abnormal & 2.9672 & 1.3323 to 6.6083 & 0.0078 \\
$\mathrm{Hb}$ & 0.5791 & 0.4585 to 0.7314 & $<0.0001$ \\
Anaemia & & & \\
$\quad$ *Normal & & & \\
Mod-severe & 3.1385 & 1.2099 to 8.1412 & 0.0187 \\
URE: CRE & & & \\
$\quad$ * Normal & & & \\
Intrarenal azotaemia & 0.1893 & 0.0602 to 0.5955 & 0.0044 \\
UREA & 3.6435 & 2.0968 to 6.3311 & $<0.0001$ \\
\hline
\end{tabular}

CRE: Creatinine. URE: Urea. OR: Odds ratio. Adj: Adjusted. Cl: Confidence Interval. $\mathrm{P}$ values less $<0.05$ were considered significant. ${ }^{*}$ Reference variable
$(<10 \mathrm{~g} / \mathrm{dL})$ and an abnormal peripheral blood smear were independently associated with a higher likelihood of developing primary $\mathrm{PPH}$.

\section{Biochemical parameters and primary PPH}

AST and ALB levels, although differed significantly between the two groups, were within normal limits of pregnancy [23]. This is similar to a study where there was significant changes in ALT but not in other liver enzymes [25]. Higher levels of AST than ALT could be accounted for from other sources of the enzyme, the heart, skeletal muscles, kidney, brain and red cells [26]. The exclusion of participants with liver infection and other chronic liver diseases could be a contributing factor for the observation of normal liver function markers amongst the study participants.

Urea and creatinine levels were higher amongst Group 1 (women with primary PPH) than in Group 2. Creatinine is expected to decline in pregnancy [23, 27], hence mild elevations during pregnancy may signify mild renal insufficiency, as opposed to that in non-pregnant women. We however did not determine the estimated glomerular filtration rate (eGFR) of participants, therefore this suggestion is not conclusive. Upon multivariate analysis, Urea and urea-to-creatinine ratio remained significant associations of primary PPH. It has been suggested that excess urea can cause an upregulation of endothelial nitric oxide through shunting of L-arginine to form guanidinosuccinic acid [28]. Consequently, this 
causes reduced endothelial adhesion of platelets due to impaired Von-Willibrand Factor (VWF) binding to platelets, therefore higher bleeding tendencies [28].

\section{Coagulopathy and primary PPH}

Measures of coagulation, PT and aPTT did not differ between the two groups and hence showed no significant association. It is expected that bleeding tendencies will correlate with abnormalities in coagulation markers. Although the current study could not demonstrate this the finding is still in line with findings that coagulation abnormalities contribute only about $1 \%$ of the causes of PPH [29], hence our observation. It is important however, to note that coagulation abnormalities cannot be ruled out. Suggestions are that a hypercoagulable state results in the second and third trimesters due to an increased synthesis and activity of coagulation factors fibrinogen, coagulation factors (F) VII, VIII, IX, X, XII, and VWF - and results in a shortened PT and aPTT [30]. This could be a contributing factor to our observation of a pseudo-normal PT and aPTT. Hence coagulation abnormalities may have been present but masked by physiological changes, and may be apparent only after pregnancy.

\section{Haematological parameters and primary PPH}

We found that increasing haemoglobin concentrations were associated with a less likelihood of primary $\mathrm{PPH}$ and that women with $[\mathrm{Hb}]<10$ (moderate-severe anaemia) were more likely to develop primary $\mathrm{PPH}$. Anaemia is responsible for $17-46 \%$ of cases of maternal deaths when combined with obstetric haemorrhage [22]. In addition, complications of $\mathrm{PPH}$, pre-term delivery and foetal growth restriction are more frequent in anaemic women [31]. In a similar study, women in Zimbabwe with an $[\mathrm{Hb}]$ of less than $12 \mathrm{~g} / \mathrm{dL}$ were 2.2 times more likely to develop PPH than non-PPH controls [18]. Researchers in Tanzania reported that $[\mathrm{Hb}]$ of $<9 \mathrm{~g} / \mathrm{dL}$ was strongly associated with bleeding in the immediate postpartum period [12]. In contrast to our findings, two previous studies reported no association between anaemia and PPH in Nigeria [32] and no difference in risk of postpartum haemorrhage between severe and moderate anaemia in Ghana [33]. These studies however relied on visual estimation, a technique known to underestimate blood loss [5, 34].

It has been hypothesized that severe anaemia may weaken uterine muscles or lower resistance to infectious diseases thus leading to $\mathrm{PPH}[35,36]$. Furthermore, decreased uterine blood flow or low uterine muscle strength may contribute to inefficient uterine contractions and contribute to blood loss, potentially mediated by iron-deficiency anaemia [37, 38]. The findings of this current study have demonstrated that the severity of anaemia places a woman at a greater risk of experiencing $\mathrm{PPH}$. It also confirms the hypothesis that severe anaemia may impair the tolerance of women to the effects of $\mathrm{PPH}$ due to their inability to endure excessive blood losses [39, 40].

The association of low haemoglobin concentrations and thus anaemia with primary PPH is consistent with our observation that an abnormal blood film was associated with a higher risk of primary PPH. It is noteworthy that the morphological appearance and number of red cells are largely affected by the haemoglobin concentration and anaemia. Furthermore, other conditions that may affect platelet function and numbers leading to an abnormal peripheral blood smear may stimulate bleeding thus leading to $\mathrm{PPH}$.

\section{Recommendations and impact on health delivery system}

Currently in Ghana, the routine antenatal laboratory tests and screening include some but not all the parameters and variables identified in the present study. While we have made progress, the current interventions are inadequate. The practicality of carrying out the extra tests proposed in the present study, including cost, capacity of the facility and the laboratory, as well as cost implications to the patient and the facility should be evaluated appropriately by the relevant authority. Accordingly, a review of antenatal policies to cater for such additional proposed tests may be warranted.

A total clinical work-up including laboratory evaluation of the independent blood variables identified in this study could help in identifying individuals at high risk or otherwise for $\mathrm{PPH}$. The will contribute to prevention, intervention or ensuring preparations for active management in the early stages.

In settings where there is lack of skilled personnel, a more preventive approach to primary $\mathrm{PPH}$ is recommended; indicators identified in the present study could be used to identify and refer high-risk individuals to better-resourced facilities to prevent maternal and neonatal death in the event of the occurrence of primary $\mathrm{PPH}$.

\section{Conclusions}

Many laboratory parameters are associated with primary $\mathrm{PPH}$, However, only a few serve as independent risk factors; increasing urea and urea-to-creatinine ratio, decreasing haemoglobin concentration $(<10 \mathrm{~g} / \mathrm{dL})$ and an abnormal peripheral blood smear were independently associated with a higher likelihood of developing primary $\mathrm{PPH}$.

\section{Limitations}

There is a limitation on the generalization of the study findings due to the consecutive sampling technique and 
the relatively smaller sample size used. Further studies using a larger sample pool in matched case-control studies are warranted. Furthermore, a representation of haemoglobin levels and the extent of blood loss in each group would better buttress the relationship between $\mathrm{Hb}$ and the extent of blood loss required for primary PPH development. However given the lack of availability of such data in Ghana, findings from this study however remain relevant and add to evidence on the subject matter.

\section{Abbreviations \\ ALB: Albumin; ALT: Alanine Aminotransferase; AMSTL: Active Management of the Third Stage of Labour; aPTT: Activated Partial Thromboplastin Time; AST: Aspartate Aminotransferase; CRE: Creatinine; EDTA: ethylene-diamine- tetra-acetic acid; ESR: Erythrocyte Sedimentation Rate; FBC: Full Blood Count; G6PD: Glucose-6-phosphate Dehydrogenase; GLOB: Globulins; KATH: Komfo Anokye Teaching Hospital; MDG: Millennium Development Goals; PPH: Postpartum haemorrhage; PT: Prothrombin time; TP: Total Protein; URE: urea; VWF: Von-Willibrand Factor.}

\section{Competing interests}

The authors hereby declare that there are no competing interests regarding this publication.

\section{Authors' contributions}

WKBAO was involved in conception of the idea, study design, interpretation of data, revision and final approval of the manuscript. DNMO was involved in conception of the idea, study design, data collection, compilation and analysis of data and drafting of the manuscript. CAT was involved in conception of the idea, study design recruitment and allocation of participants during data collection, revision and final approval of the manuscript. OOA was involved in data collection, interpretation of data, literature search and drafting of manuscript. All authors read and approved the final manuscript.

\section{Acknowledgements}

Our gratitude goes to the nurses and staff at the labour ward, ward A2, KATH and to Margaret Rosa-Puckey and Asamoah Kyei, for their contribution in patient recruitment. We appreciate Mr. David Sambien and all staff of the malaria research laboratory, KATH. Also to Dr. Richard Kobina Dadzie Ephraim of UCC, Dr. Christian Obirikorang of SMS/KNUST and James Osei Yeboah of UHAS, Ho, Ghana.

\section{Author details}

${ }^{1}$ Department of Molecular Medicine, School of Medical Sciences (SMS), Kwame Nkrumah University of Science and Technology (KNUST), Kumasi, Ghana. ${ }^{2}$ Department of Obstetrics and Gynaecology, Komfo Anokye Teaching Hospital (KATH)/SMS, KNUST, Kumasi, Ghana.

\section{Received: 15 February 2014 Accepted: 13 January 2016} H.

\section{References}

1. WHO: Trends in maternal mortality: 1990 to 2013. In: Estimates by WHO, UNICEF, UNFPA, The World Bank and the United Nations Population Division. Geneva, Switzerland: World health organization; 2014.

2. Maternal mortality ratio (modeled estimate, per 100,000 live births) [http://data.worldbank.org/indicator/SH.STA.MMRT]

3. Rath WH. Postpartum hemorrhage-update on problems of definitions and diagnosis. Acta Obstet Gynecol Scand. 2011;90(5):421-8.

4. Naz H, Sarwar I, Fawad A, Nisa AU. Maternal morbidity and mortality due to primary PPH: experience at Ayub Teaching Hospital Abbottabad. J Ayub Med College Abbottabad. 2008;20(2):59-65.

5. Maternity-Prevention, Early Recognition and Management of Postpartum Haemorrhage (PPH). In. North Sydney: Department of Health, New South Wales; 2010: 16.
6. El-Refaey H, Rodeck C. Post-partum haemorrhage: definitions, medical and surgical management. A time for change. Br Med Bull. 2003;67:205-17.

7. Magann EF, Evans S, Hutchinson M, Collins R, Howard BC, Morrison JC. Postpartum hemorrhage after vaginal birth: an analysis of risk factors. South Med J. 2005;98(4):419-22.

8. Al-Zirqi I, Vangen S, Forsen L, Stray-Pedersen B. Prevalence and risk factors of severe obstetric haemorrhage. BJOG. 2008;115(10):1265-72.

9. Oyelese $Y$, Ananth CV. Postpartum hemorrhage: epidemiology, risk factors, and causes. Clin Obstet Gynecol. 2010;53(1):147-56.

10. Magann EF, Doherty DA, Briery CM, Niederhauser A, Chauhan SP, Morrison JC. Obstetric characteristics for a prolonged third stage of labor and risk for postpartum hemorrhage. Gynecol Obstet Invest. 2008;65(3):201-5.

11. Combs CA, Murphy EL, Laros Jr RK. Factors associated with postpartum hemorrhage with vaginal birth. Obstet Gynecol. 1991;77(1):69-76.

12. Kavle JA, Stoltzfus RJ, Witter F, Tielsch JM, Khalfan SS, Caulfield LE. Association between anaemia during pregnancy and blood loss at and after delivery among women with vaginal births in Pemba Island, Zanzibar, Tanzania. J Health Popul Nutr. 2008;26(2):232-40.

13. Sheiner E, Sarid L, Levy A, Seidman DS, Hallak M. Obstetric risk factors and outcome of pregnancies complicated with early postpartum hemorrhage: a population-based study. J Matern Fetal Neonatal Med. 2005;18(3):149-54.

14. Marchant S, Alexander J, Thomas P, Garcia J, Brocklehurst P, Keene J. Risk factors for hospital admission related to excessive and/or prolonged postpartum vaginal blood loss after the first $24 \mathrm{~h}$ following childbirth. Paediatr Perinat Epidemiol. 2006;20(5):392-402.

15. Ramanathan G, Arulkumaran S. Postpartum hemorrhage. J Obstet Gynaecol Can. 2006;28(11):967-73.

16. Maughan KL, Heim SW, Galazka SS. Preventing postpartum hemorrhage: managing the third stage of labor. Am Fam Physician. 2006;73(6):1025-8.

17. Prata N, Hamza S, Bell S, Karasek D, Vahidnia F, Holston M. Inability to predict postpartum hemorrhage: insights from Egyptian intervention data. BMC Pregnancy Childbirth. 2011;11:97.

18. Tsu VD. Postpartum haemorrhage in Zimbabwe: a risk factor analysis. Br J Obstet Gynaecol. 1993:100(4):327-33.

19. Geller SE, Goudar SS, Adams MG, Naik VA, Patel A, Bellad MB, et al. Factors associated with acute postpartum hemorrhage in low-risk women delivering in rural India. Int J Gynaecol Obstet. 2008;101(1):94-9.

20. Lertbunnaphong $T$, Leetheeragul J, Thitadilok W. Risk factors of primary postpartum hemorrhage in Siriraj Hospital. Siriraj Med J. 2010;62:5. unpaginated.

21. Gulmezoglu AM, Hofmeyr GJ. Prevention and treatment of postpartum haemorrhage. In: MacLean AB, Neilson J, editors. Maternal Morbidity and Mortality. London: RCOGPress; 2002. p. 434.

22. Carroli G, Cuesta C, Abalos E, Gulmezoglu AM. Epidemiology of postpartum haemorrhage: a systematic review. Best Pract Res Clin Obstet Gynaecol. 2008;22(6):999-1012

23. Tran HA. Biochemical tests in pregnancy. Australian Prescriber. 2005:28(4):99.

24. DeMaeyer EM, Dallman P, Gurney JM, Hallberg L, Sood SK, Srikantia SG. Preventing and controlling iron deficiency anemia through primary health care. A guide for Health administrators and program managers. Geneva, Switzerland: World Health Organization; 1989. p. 26.

25. Puri M, Patra S, Singh P, Malhotra N, Trivedi SS, Sharma S, et al. Factors influencing occurrence of postpartum haemorrhage in pregnant women with hepatitis $\mathrm{E}$ infection and deranged coagulation profile. Obstetric Med. 2011:4:108-12.

26. Cheesbrough M. District Laboratory Practice in Tropical Countries. vol. 2nd ed. New York: Cambridge University Press; 2006. p. 329-33.

27. Abbassi-Ghanavati M, Greer LG, Cunningham FG. Pregnancy and laboratory studies: a reference table for clinicians. Obstet Gynecol. 2009;114(6):1326-31.

28. Collins PW, Thachil J, Toh C-H. Acquired Coagulation Disorders. In: Hoffbrand AV, Catovsky D, Tuddenham EGD, Green AR, editors. Postgraduate Haematology. 6th ed. Oxford, U.K: Blackwell Publishing; 2011. p. 839-59.

29. Anderson J, Etches D, Smith D: Postpartum haemorrhage. In Damos J R Eisinger S H, eds. Kansas: American Academy of Family Physicians; 2000.

30. Ahonen J, Stefanovic V, Lassila R. Management of post-partum haemorrhage. Acta Anaesthesiol Scand. 2010;54(10):1164-78.

31. Fareh Ol, Rizk DE, Thomas L, Berg B. Obstetric impact of anaemia in pregnant women in United Arab Emirates. J Obstet Gynaecol. 2005:25(5):440-4.

32. Selo-Ojeme DO, Okonofua FE. Risk factors for primary postpartum haemorrhage. A case control study. Arch Gynecol Obstet. 1997;259(4): 179-87. 
33. Geelhoed D, Agadzi F, Visser L, Ablordeppey E, Asare K, O'Rourke P, et al. Maternal and fetal outcome after severe anemia in pregnancy in rural Ghana. Acta Obstet Gynecol Scand. 2006;85(1):49-55.

34. Turpin CA, Osakunor DNM, Owiredu WKBA. Accuracy of Blood Loss Determination after Vaginal Delivery: Visual Estimation versus Calibrated Measurement. BJMMR. 2015;6(11):1121-7.

35. Rush D. Nutrition and maternal mortality in the developing world. Am J Clin Nutr. 2000;72(1 Suppl):212S-40S.

36. Bergstrom S. Infection-related morbidities in the mother, fetus and neonate. J Nutr. 2003;133(5 Suppl 2):1656S-60S.

37. Brotanek V, Hendricks $\mathrm{CH}$, Yoshida T. Changes in uterine blood flow during uterine contractions. Am J Obstet Gynecol. 1969;103(8):1108-16.

38. Ulmer HU, Goepel E. Anemia, ferritin and preterm labor. J Perinat Med. 1988;16(5-6):459-65.

39. Allen LH. Anemia and iron deficiency: effects on pregnancy outcome. Am J Clin Nutr. 2000;71(5 Suppl):1280S-4S.

40. Alauddin M. Maternal mortality in rural Bangladesh: the Tangail District. Stud Fam Plann. 1986;17(1):13-21.

Submit your next manuscript to BioMed Central and we will help you at every step:

- We accept pre-submission inquiries

- Our selector tool helps you to find the most relevant journal

- We provide round the clock customer support

- Convenient online submission

- Thorough peer review

- Inclusion in PubMed and all major indexing services

- Maximum visibility for your research

Submit your manuscript at www.biomedcentral.com/submit
Biomed Central 